\title{
Stringent numerical test of the Poisson distribution for finite quantum integrable Hamiltonians
}

\author{
A. Relaño, ${ }^{1}$ J. Dukelsky, ${ }^{2}$ J. M. G. Gómez, ${ }^{1}$ and J. Retamosa ${ }^{1}$ \\ ${ }^{1}$ Departamento de Física Atómica, Molecular y Nuclear, Universidad Complutense de Madrid, E-28040 Madrid, Spain \\ ${ }^{2}$ Instituto de Estructura de la Materia, CSIC, Serrano 123, 28006 Madrid, Spain
}

(Received 13 November 2003; revised manuscript received 4 May 2004; published 19 August 2004)

\begin{abstract}
Using a class of exactly solvable models based on the pairing interaction, we show that it is possible to construct integrable Hamiltonians with a Wigner distribution of nearest-neighbor level spacings. However, these Hamiltonians involve many-body interactions and the addition of a small integrable perturbation very quickly leads the system to a Poisson distribution. Besides this exceptional case, we show that the accumulated distribution of an ensemble of random integrable two-body pairing Hamiltonians is in perfect agreement with the Poisson limit. These numerical results for quantum integrable Hamiltonians provide a further empirical confirmation of the work of Berry and Tabor in the semiclassical limit.
\end{abstract}

DOI: $10.1103 /$ PhysRevE.70.026208

PACS number(s): 05.45.Mt, 02.30.Ik

The concept of quantum chaos still lacks a clear definition. The main ideas in this field have been obtained using the semiclassical approximation for quantum systems having a classical analog. In a seminal paper, Bohigas et al. [1] conjectured that the fluctuation properties of generic quantum systems, which in the classical limit are fully chaotic, coincide with those of random matrix theory (RMT). This conjecture is strongly supported by experimental data, numerical calculations, and analytical work based on semiclassical arguments. For a generic quantum integrable system, Berry and Tabor [2] showed that under very general conditions the spectral fluctuations in the semiclassical limit are well described by the Poisson statistics; i.e., the successive energy levels are uncorrelated.

The analysis of spectral fluctuations provides an essential tool in the study of quantum chaos. Moreover, as RMT was introduced to explain the fluctuation properties of manybody systems like the atomic nucleus, it is usually considered that the level statistics establishes a link between manybody systems and the semiclassical picture. Actually, if the semiclassical limit is not valid, comparison of the system spectral fluctuations with those predicted by RMT is the main criterion to decide whether the system dynamic is regular or chaotic. When spectral fluctuations fall very near the RMT predictions the quantum system is considered fully chaotic. On the contrary, if they follow closely enough the Poisson statistic, the system is considered regular. Therefore, the concepts of chaotic and regular quantum systems are not well established since they rely on results that have only been checked in the semiclassical limit.

The concept of integrability in classical mechanics was well defined after the work of Liouville in the 19th century. A classical Hamiltonian system is integrable if it has a complete set of independent integrals of motion commuting with respect to the Poisson brackets. The total number of integrals of motion should be half of the dimension of the phase space to assure completeness. In quantum mechanics, the concept of integrability is usually derived from an extension of the Liouville definition. A quantum system is said to be integrable if it is possible to define a complete set of Hermitian operators, the integrals of motion, which commute among each other. However, this definition has some deficiencies and ambiguities. Various attempts to clarify the characteristics of these integrals of motions-mainly, their functional independene-have not produced irrefutable answers (see, for instance, [3-5] and references therein). In this work we shall use the following definition: a quantum system is said to be integrable if a set of as many commuting Hermitian operators as quantum degrees of freedom can be explicitly given, and the Hamiltonian can be expressed as a function of these operators [4]. This criterion, relying in the algebraic structure of quantum mechanics, does not directly refer to classical mechanics. Since this definition requires that a "complete" set of quantum integrals of motion be explicitly given, it assures the existence of a common basis of eigenstates labeled by the eigenvalues of the integrals of motion. Moreover, the system is exactly solvable if the complete set of eigenstates can be found by algebraic methods. In this sense, the previous definition of quantum integrability is closely related to exact solvability. We will use this criterion to test some accepted properties of quantum integrable systems, like the Berry-Tabor conjecture, without taking into account the classical limit.

The level statistics of quantum integrable models has been analyzed in condensed-matter physics as well as in nuclear physics. Poilblanc et al. [6] studied spectral fluctuations by finding the energy spectrum for several one-dimensional finite lattice models, like the Heisenberg model, the $t$ - $J$ model, and the Hubbard model. Alhassid and Novoselsky [7] studied the quantal fluctuations displayed by the energy levels in the interacting boson model of nuclei. Recently, the realization of a Poisson distribution has been suggested as a detector of new integrable quantum chiral Potts models [8]. In each case, the integrable Hamiltonian is parameter independent or it depends on a single free parameter like in the Hubbard model (the on-site repulsion $U$ ). The study of spectral fluctuations has been carried out diagonalizing a definite Hamiltonian in the largest possible Hilbert space. In all cases of integrable quantum models, it was verified that the histogram of the near-neighbor level spacing could be well fitted by a Poisson distribution. These results provide numerical support for the Berry-Tabor semiclassical demonstration [6-8], but the quality of the statistics is poor due to the small number of energy levels entering in the histogram. This is in contrast 
with numerical testing of the Bohigas-Giannoni-Schmit [1] conjecture, assigning a Wigner distribution to any nonintegrable quantum Hamiltonian, in which large ensembles of two-body random Hamiltonians were considered (for a review see [9]). More recently, Benet et al. [10] have studied an ensemble of integrable bosonic Hamiltonians whose members display GOE- or GUE-like spectra with probability 1. This anomalous behavior can be explained in terms of semiclassical mechanics. Despite the fact that the systems under consideration were integrable (in the semiclassical sense), the periodic orbits that fulfill the Einstein-BrillouinKeller (EBK) quatization condition explore huge regions of phase space; i.e., they mimic a typical chaotic motion. Thus, it is reasonable to find random matrix spectral fluctuations. Therefore, the whole ensemble can be considered an anomalous exception of the Berry-Tabor conjecture.

Trying to get more insight into these ideas, we study the level statistic of a class of quantum integrable models: the Richardson-Gaudin models. They are based on the pairing interaction [11] and have a large number of free parameters which can be selected randomly. In particular, we will study the rational model for which the quantum invariants have the form

$$
R_{i}=K_{i}^{0}+2 g \sum_{j(\neq i)} \frac{1}{\eta_{i}-\eta_{j}}\left\{\frac{1}{2}\left(K_{i}^{+} K_{j}^{-}+K_{i}^{-} K_{j}^{+}\right)+K_{i}^{0} K_{j}^{0}\right\},
$$

where $i$ labels the $\Omega$ levels of a single-particle basis, $\eta_{i}$ are $\Omega$ free real parameters, and $g$ is the paring strength. The operators $K^{+}, K^{-}$, and $K^{0}$ are the $\mathrm{SU}(2)$ generators of the pair algebra in level $i$ :

$$
K_{i}^{0}=\frac{1}{2}\left(a_{i}^{\dagger} a_{i}+a_{i}^{\dagger} a_{i}^{-}-1\right), \quad K_{i}^{+}=\frac{1}{2} a_{i}^{\dagger} a_{i}^{\dagger}=\left(K_{i}^{-}\right)^{\dagger} .
$$

The operator $K_{i}^{+}$creates a pair of particles in time reversal states in the double-degenerated level $i$. The three generators close the commutation algebra su(2):

$$
\left[K_{l}^{0}, K_{l^{\prime}}^{+}\right]=\delta_{l l^{\prime}} K_{l}^{+}, \quad\left[K_{l}^{+}, K_{l^{\prime}}^{-}\right]=2 \delta_{l l^{\prime}} K_{l}^{0} .
$$

Based on this commutation algebra, it is straightforward to check that the operators (1) commute with one another for arbitrary values of $g$ and the set of $\Omega$ parameters $\eta$. The rational model, as well as other models, is fully integrable and exactly solvable (for the exact eigenstates of these models see [11]).

Once the free parameters inside the $R_{i}$ operators are fixed, it is possible to find their complete set of common eigenvalues $r_{i}^{\alpha}$ and eigenvectors $\left|\psi_{\alpha}\right\rangle$. Any function of the $R_{i}$ operators defines a valid integrable Hamiltonian. In particular, linear combinations of the $R_{i}$ operators produce quite general pairing Hamiltonians

$$
\begin{aligned}
H & =\sum_{i} \varepsilon_{i} R_{i} \\
& =\sum_{i} \varepsilon_{i} K_{i}^{0}+g \sum_{j \neq i)} \frac{\varepsilon_{1}-\varepsilon_{j}}{\eta_{i}-\eta_{j}}\left\{\frac{1}{2}\left(K_{i}^{+} K_{j}^{-}+K_{i}^{-} K_{j}^{+}\right)+K_{i}^{0} K_{j}^{0}\right\} .
\end{aligned}
$$

Contrary to most of the integrable Hamiltonians cited above, this class of integrable Hamiltonians depends on $(2 \Omega+1)$ free, real, and independent parameters; combinations of higher-rank quantum invariants would give larger sets of free parameters. Within this very large parameter space it is worth exploring the possibility of finding chaotic spectra, contradicting the Berry-Tabor hypothesis. Therefore the usual distinction between chaos and regularity by means spectral statistics would become doubtful.

To shed some light on this question we have tried to fit several chaotic spectra using the class of Hamiltonians (4) for different values of $\Omega$ and different particle pair numbers $N$. These chaotic spectra were obtained by diagonalizing a random matrix with the appropriate dimension. The dimension of the Hilbert space for a system with $\Omega$ levels and $N$ fermion pairs is given by

$$
D=\left(\begin{array}{l}
\Omega \\
N
\end{array}\right) .
$$

Thus, it is usually much larger than the number of Hamiltonian parameters $2 \Omega+1$; therefore, it is impossible to obtain an exact replica of the actual random matrix spectrum, and we can only get an approximation to this spectrum. In all the cases the best parameter set leads to a Hamiltonian whose spectrum shows Poisson level fluctuations, as predicted by Berry and Tabor.

Nevertheless, as we have commented above, it is still possible to consider more general Hamiltonians using the $R_{i}$ operators. These Hamiltonians involve many-body forces represented by combinations of higher-rank $R$ operators. Knowing the dimension $D$ of the Hilbert space for a system with $\Omega$ levels and $N$ fermion pairs, we propose the following class of integrable Hamiltonians with many body forces:

$$
H=\sum_{i_{1}>i_{2}>\cdots>i_{N}} \varepsilon_{i_{1}, i_{2}, \ldots, i_{N}} R_{i_{1}} R_{i_{2}} \cdots R_{i_{N}} .
$$

If we fix the $\eta_{i}$ parameters and the value of $g$ inside the $R_{i}$ operators entering in Eq. (5), the number of free parameters $\varepsilon_{i_{1}, i_{2}, \ldots, i_{N}}$ in the Hamiltonian is precisely equal to the dimension of the Hilbert space $D$. Therefore, the $d$ eigenvalues of a nonintegrable pairing Hamiltonian with a typical chaotic spectrum can be exactly fitted with the Hamiltonian (5) by solving a linear set of equations for the $D$ unknowns $\varepsilon_{i_{1}, i_{2}, \ldots, i_{N}}$

We have considered several cases with $(\Omega, N)=(10,6)$, $(11,6),(12,6)$, and $(13,6)$; the corresponding dimensions of the Hamiltonian matrices are $D=210,462,924$, and 1716, respectively. In each case we have been able to fit a chaotic spectra with the appropriate dimensionality obtaining an exception to the Berry-Tabor result. In other words, we have been able to obtain spectral fluctuations of Wigner-Dyson 
type at all energy scales for quantum integrable Hamiltonians. Actually, exceptions to this rule were already known: for example, Crehan [12] proved that any spectral sequence obeying a certain growth restriction is the quantum spectrum of an equivalence class of classically integrable nonlinear oscillators. Our result, however, is quite more enlightening because it provides an example of a full and realistic quantum system where the semiclassical result of Berry and Tabor does not apply.

Our findings raise the question of how stable are these solutions against small perturbations of the Hamiltonian parameters within the parameter space and whether finite-size effects may affect these properties. To study these two points the Hamiltonians obtained in the previous fit are perturbed as follows. Each parameter $\varepsilon_{i_{1}, i_{2}, \ldots, i_{N}}$ in Eq. (5) is replaced by

$$
\varepsilon \rightarrow \varepsilon^{\prime}=\varepsilon(1+\phi \lambda),
$$

where $\lambda$ stands for the perturbation strength and $\phi$ is a phase chosen at random. Notice that the new Hamiltonian is also a combination of $R_{i}$ operators and therefore is integrable.

Before we proceed to analyze the spectral fluctuations of the perturbed Hamiltonians, their spectra must be unfolded. For any quantum system the level density $\rho(E)$ can be separated into a smooth part $\overline{\rho(E)}$ and a fluctuating part $\widetilde{\rho(E)}$. The former gives the main trend of the level density and the later characterizes the spectral fluctuations. Similarly, the cumulative level density, which gives the true number of levels up to energy $E$,

$$
N(E)=\int_{-\infty}^{E} d E^{\prime} \rho\left(E^{\prime}\right)
$$

can be separated into a smooth part $\overline{\rho(E)}$ and a fluctuating $\widetilde{\rho(E)}$ part, i.e.,

$$
N(E)=\overline{N(E)}+\widetilde{N(E)} .
$$

Actually, level fluctuation amplitudes are modulated by the value of the mean level density $\rho(E)$; therefore, to compare the fluctuations of different systems or even the fluctuations of different parts of the same spectrum, the level density smooth behavior must be removed. This is done by means of a transformation, called unfolding [13], which consists of mapping the energy levels $E_{i}$ onto dimensionless $\zeta_{i}=\overline{N(E)}$. Then, the nearest-neighbor spacing sequence is defined by

$$
s_{i}=\zeta_{i+1}-\zeta_{i}, \quad i=1, \ldots, N-1 .
$$

For the unfolded levels $\bar{\rho}(\epsilon)=1$ and $\langle s\rangle=1$. In general this is a difficult task for systems where an analytical expression for the mean level density is not known. This is the case of the Hamiltonian ensembles introduced in the present paper. Therefore, in order to obtain a good approximation to $\overline{N(E)}$ we have performed a least-squares fit to the Chebyshev polynomials.

The spectral properties of the perturbed Hamiltonians can be analyzed by means of different statistics. The most simple is the nearest-neighbor spacing distribution $P(s)$. It allows us to quantify the chaoticity of the system in terms of a single parameter $\omega$ by fitting the $P(s)$ histogram to the Brody dis-

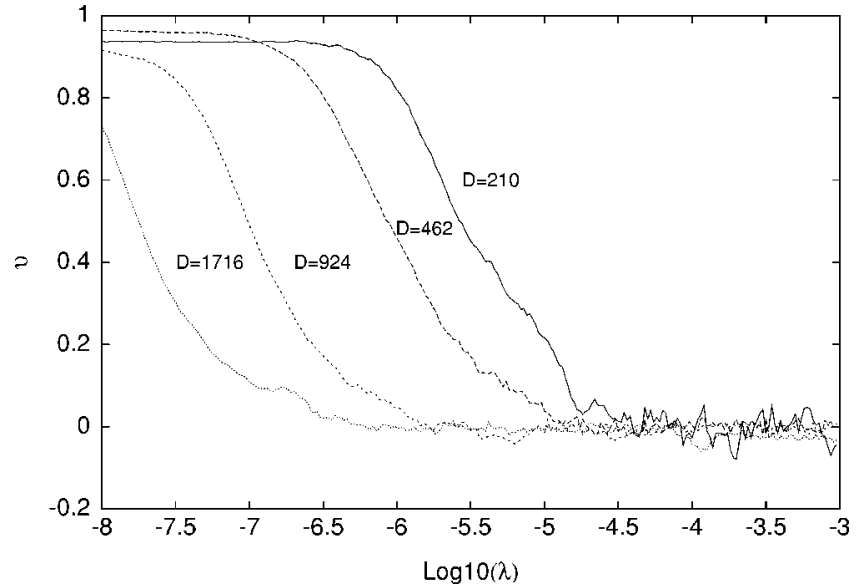

FIG. 1. Variation of the Brody parameter $\omega$ as a function of the perturbation strength $\lambda$ for $(\Omega, N)=(10,6),(11,6),(12,6)$, and $(13,6)$.

tribution $P(s, \omega)$ [14] using a least-squares method. The resulting distribution interpolates between the Poisson limit $(\omega=0)$ and the Wigner limit $(\omega=1)$. It would be also possible to analyze the behavior of the eigenstates as a function of the perturbation strength. This could be done calculating the information entropy $I_{H}$ or the localization length $l_{H}[15]$. However, we can advance that the Hamiltonian eigenstates will remain unaltered under the influence of the perturbation (6) which is defined on the coefficients of the combination of integrals of motion but does not modify them. Actually, for any integrable system the eigenstates are completely defined by the quantum integrals of motion, and the Hamiltonian can be expressed as a function of these operators. Therefore, all Hamiltonians obtained using different functions of a given complete set of integrals of motion will have the same eigenstates.

Figure 1 displays in a semilogarithmic scale the Brody parameter $\omega$ as a function of the perturbation strength $\lambda$ for the four $(\Omega, N)$ examples we have considered before. In all cases a very small perturbation is enough to drive the system to the Poisson limit. For the smallest system we considered ( $D=210)$ a perturbation $\lambda \sim 10^{-5}$ is enough to obtain Poissonian spectral fluctuations, while for the largest system ( $D$ $=1716$ ) three orders of magnitude less are required. Clearly, the trend is that larger systems require smaller perturbations.

In the light of these results we conclude that it is necessary to consider integrable Hamiltonians with many-body interactions in order to obtain a chaoticlike energy spectrum. However, small perturbations within the integrable space of parameters restore the Poissonian-like spectrum. We conjecture that for very large $\Omega$ and $N$ values a chaotic spectra would correspond to isolated points in the parameter space and that a infinitesimal perturbation within this space would immediately drive the system to a Poisson distribution.

In some cases it is possible to introduce a suitable random matrix model that describes the behavior of spectral fluctuations as the system evolves through parameter space. This has already been done for the metal-insulator transition or for the order-disorder transition in quantum Hall systems [16]. However, in the present case, this approach seems to be more 


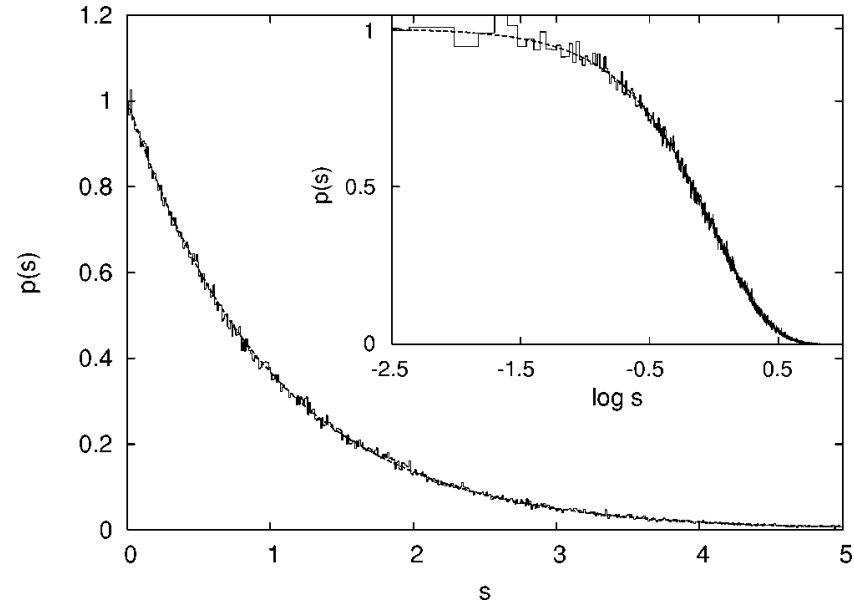

FIG. 2. Nearest-neighbor spacing distribution $P(s)$ for 200 TBPRE members. The dashed curve corresponds to the Poisson limit.

complicated since the "Wigner phase" corresponds to isolated points in parameter space.

In view of the previous results, one would expect that a more physically sound family of pairing Hamiltonians with two-body forces like Eq. (4) should give rise to a clean Poisson level statistics. In order to verify the correctness of this statement we have studied an ensemble of Hamiltonians (4), which we shall call a "two-body pairing random ensemble" (TBPRE). We selected the case of $(\Omega, N)=(13,6)$, corresponding to largest dimension $D=1716$. For the sake of simplicity, the $\eta_{i}$ parameters and the strength $g$ were given fix values and the $\varepsilon_{i}$ coefficients were selected randomly. The quantum invariants $R_{i}(1)$ are independent of the $\varepsilon_{i}$ parameters and, thus, their corresponding eigenvalues stay the same for the whole ensemble. In our calculations we have used 200 ensemble members.

The short- and long-range spectral correlations of this ensemble have been analyzed by means of the usual level statistics distribution $P(s)$ and by the rigidity $\Delta_{3}(L)$, respectively. In the Poisson limit, characteristic of a regular system, the nearest-neighbor spacing distribution behaves as $P^{\text {Poisson }}(s)=\exp (-s)$ and $\Delta_{3}^{\text {Poisson }}(L)=L / 15$. Figure 2 compares the nearest-neighbor distribution $P(s)$, calculated nu-

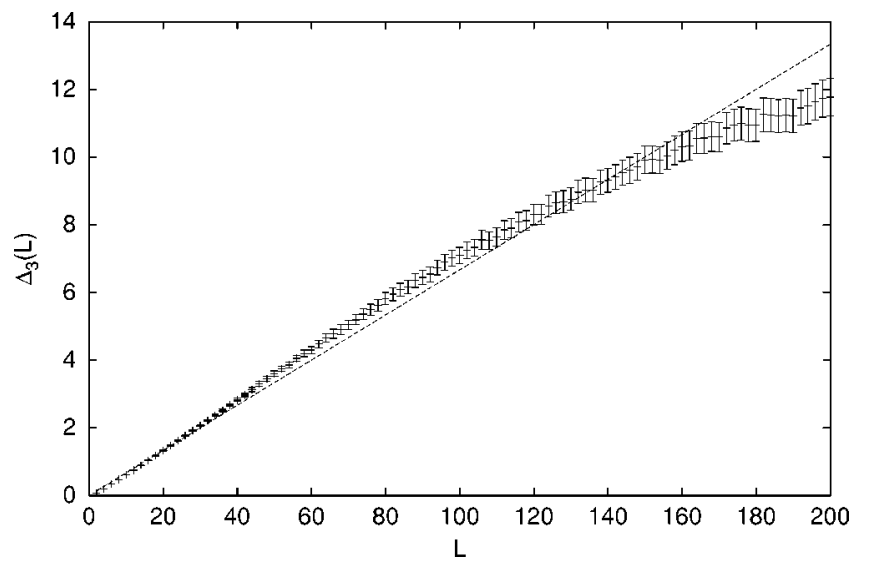

FIG. 3. $\overline{\Delta_{3}(L)}$ statistic calculated for 200 TBPRE members. The dashed line represents the Poisson values.

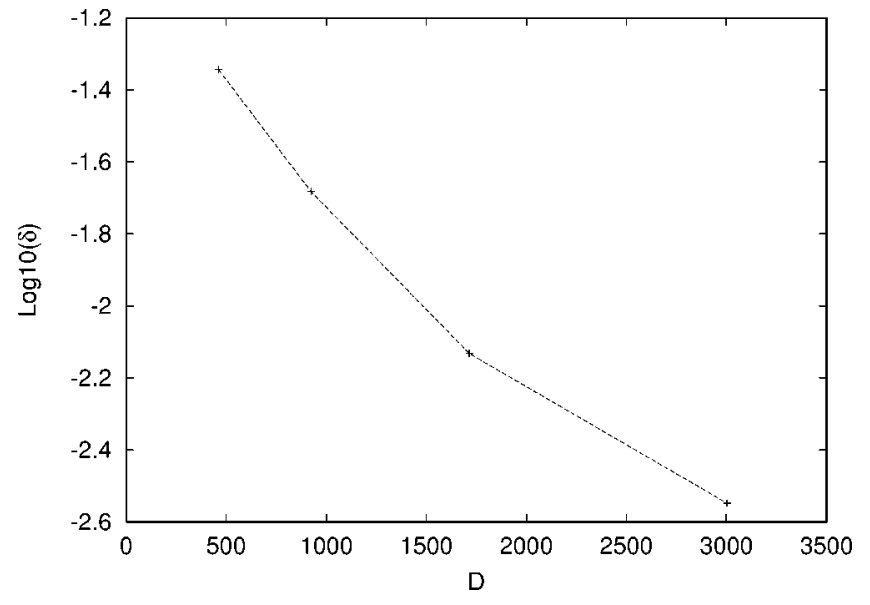

FIG. 4. Logarithmic plot of the average quadratic distance $\delta$ between the accumulated nearest spacing distribution obtained from the 200 TBPRE members and the Poisson limit, given by $n^{\text {Poisson }}(s)=1-\exp (-s)$.

merically for our ensemble with the expected Poisson limit. We present the results in normal as well as in semilogarithmic scales to enlarge the small spacing region, which has been shown to present some deviations from the Poisson limit [17]. It can be seen that the histogram and the theoretical curve match perfectly. Figure 3 shows the calculated $\Delta_{3}(L)$ and compares it to the Poisson limit $L / 15$. The agreement is almost perfect up to $L \simeq 40$; for larger $L$ values the $\Delta_{3}$ shows a slight upbending from the Poisson straight line. The calculation of the rigidity is quite sensitive to the unfolding procedure. When the mean level density is not accurately enough known, the unfolding method will introduce accumulated errors that eventually give rise to a spurious increase of the $\Delta_{3}(L)$ for large $L$ values [18]. The results shown in these two figures make it possible to conclude that the TBPRE spectral fluctuations are very well described by the Poisson statistic. Actually, Figs. 2 and 3 constitute the most precise numerical verification of the Berry-Tabor theoretical proof due to the fact that we were able to accumulate statistics by using an ensemble of random integrable Hamiltonians (TBPRE), which, to the best of our knowledge, it would not be possible for any other integrable model.

To support in a more precise way this conclusion we consider again the $p(s)$ statistics. As this statistics is less sensitive to the unfolding procedure than the $\Delta_{3}$, small deviations from the theoretical Poisson prediction can be connected to the actual characteristics of the system dynamics. To avoid any effects related to the bin size in the $P(s)$ histogram, we will use the accumulated nearest-neighbor spacing distribution $I(s)=\int_{0}^{s} P\left(s^{\prime}\right) d s^{\prime}$, which in Poisson limit is given by $I^{P o i s s o n}(s)=1-\exp (-s)$. We define a "distance" between the calculated $n(s)$ distribution and the Poisson limit as

$$
\delta^{2}=\int_{0}^{\infty}\left|n(s)-n^{\text {Poisson }}(s)\right|^{2} d s .
$$

We consider four TBPRE with $(\Omega, N)=(11,6),(12,6)$, $(13,6)$, and $(14,6)$. The matrix dimensions for these ensembles are $D=462,924,1716$, and 3003 , respectively. In 
order to have approximately $2 \times 10^{5}$ spacings in each of the four ensembles, different numbers of members were chosen for each one of them. Figure 4 shows the logarithm of the average distance $\delta$ as a function of the matrix dimensionality $D$. The most relevant observed feature is that $\delta$ decreases by an order of magnitude as the dimensionality increases from $D=462$ to $D=3003$. Moreover, the smooth and monotonous decrease of this function suggests that it goes to zero in the large- $D$ limit.

Summarizing, the use of a family of fully integrable and exactly solvable pairing models with a large number of free parameter which can be selected at random allowed us to perform several stringent tests of the Berry-Tabor semiclassical proof. Based on the numerical results obtained, we conclude that quantum integrable systems indeed follow a Poisson distribution of nearest-neighbor level spacings for large enough systems. Exceptions to this rule can be found, but we showed that they are isolated solutions of high-rank Hamiltonians and that they quickly decay to a Poisson distribution with an infinitesimal integrable perturbation.

We thank O. Bohigas, P. Leboeuf, and G. Sierra for useful discussions. This work was supported by Grant Nos. BFM2003-05316-C02-02 and BFM2000-0600.
[1] O. Bohigas, M. J. Giannoni, and C. Schmit, Phys. Rev. Lett. 52, 1 (1984).

[2] M. V. Berry and M. Tabor, Proc. R. Soc. London, Ser. A 356, 375 (1977).

[3] St. Weigert, Physica D 56, 107 (1992).

[4] S. Weigert and G. Muller, Chaos, Solitons Fractals 5, 1419 (1995).

[5] W. M. Zhang and D. H. Feng, Phys. Rep. 252, 1 (1995).

[6] D. Poilblanc, T. Ziman, J. Bellissard, F. Mila, and G. Montambaux, Europhys. Lett. 22, 537 (1993).

[7] Y. Alhassid and A. Novoselsky, Phys. Rev. C 45, 1677 (1992).

[8] J. Ch. Angles d'Auriac, J. M. Maillard, and C. M. Viallet, e-print cond-mat/0205101.

[9] V. K. B. Kota, Phys. Rep. 347, 223 (2001).

[10] L. Benet, F. Leyvraz, and T. H. Seligman, Phys. Rev. E 68,
045201(R) (2003).

[11] J. Dukelsky, C. Esebbag, and P. Schuck, Phys. Rev. Lett. 87, 066403 (2001).

[12] P. Crehan, J. Phys. A 28, 6389 (1995).

[13] F. Haake, Quantum Signatures of Chaos (Springer-Verlag, Berlin, 2001).

[14] T. A. Brody, J. Flores, J. B. French, P. A. Mello, A. Pandey, and S. S. M. Wong, Rev. Mod. Phys. 53, 385 (1981).

[15] F. M. Izrailev, Phys. Rep. 196, 299 (1990).

[16] P. Shukla, Phys. Rev. E 62, 2098 (2000).

[17] G. Casati, B. V. Chirikov, and I. Guarneri, Phys. Rev. Lett. 54, 1350 (1985).

[18] J. M. G. Gómez, R. A. Molina, A. Relaño, and J. Retamosa, Phys. Rev. E 66, 036209 (2002). 